\title{
Pneumothorax, CTCAE
}

National Cancer Institute

\section{Source}

National Cancer Institute. Pneumothorax, CT CAE. NCI Thesaurus. Code C58201.

A disorder characterized by abnormal presence of air in the pleural cavity resulting in the collapse of the lung. 